\title{
Serosurveillance among COVID-19 Cases in Ahmedabad Using SARS-COV2 IgG
} Antibodies

\author{
Om Prakash ${ }^{1}$, Bhavin Solanki ${ }^{2}$, Jay Sheth ${ }^{3}$, Daxa Maitrak ${ }^{4}$, Mina Kadam ${ }^{5}$, Sheetal Vyas ${ }^{6}$, Aparajita Shukla7, \\ Hemant Tiwari ${ }^{8}$
}

${ }^{1}$ IAS, Deputy Municipal Commissioner, Ahmedabad Municipal Corporation; ${ }^{2}$ Medical Officer of Health, Ahmedabad Municipal Corporation; ${ }^{3}$ Associate Professor, Department of Community Medicine, AMC MET Medical College, Ahmedabad, Gujarat 380008; ${ }^{4}$ Deputy Health Officer, Ahmedabad Municipal Corporation; ${ }^{5}$ Professor \& Head, Department of Microbiology, AMC MET Medical College, Ahmedabad, Gujarat 380008; ${ }^{6}$ Professor \& Head, Community Medicine, AMC MET Medical College, Ahmedabad, Gujarat 380008; 7 Professor \& Head, Department of Community Medicine, Smt. Nathiba Hargovandas Lakhmichand Municipal Medical College, Ahmedabad, Gujarat; ${ }^{8}$ Assistant Professor Statistics, Community Medicine, Smt. Nathiba Hargovandas Lakhmichand Municipal Medical College, Ahmedabad, Gujarat

\begin{tabular}{|c|c|c|c|c|c|c|c|c|}
\hline Abstract & Introduction & Methodology & Results & Conclusion & References & Citation & \multicolumn{2}{|c|}{ Tables / Figures } \\
\hline \multicolumn{9}{|c|}{ Corresponding Author } \\
\hline \multicolumn{8}{|c|}{$\begin{array}{l}\text { Dr. Jay K. Sheth, Associate Professor, Department of Community Medicine, AMC MET Medical College, } \\
\text { Maninagar, Ahmedabad } \\
\text { E Mail ID: jayksheth@yahoo.com }\end{array}$} & 回部回 \\
\hline
\end{tabular}

\section{Citation}

Prakash O, Solanki B, Sheth J, Maitrak D, Kadam M, Vyas S, Shukla A, Tiwari H. Serosurveillance among COVID-19 Cases in Ahmedabad Using SARS-COV2 IgG Antibodies. Indian J Comm Health. 2021;33(2):351-356. https://doi.org/10.47203/IJCH.2021.v33i02.022

Source of Funding: Nil Conflict of Interest: None declared

\section{Article Cycle}

Received: 04/04/2021; Revision: 11/05/2021; Accepted: 29/05/2021; Published: 30/06/2021

This work is licensed under a Creative Commons Attribution 4.0 International License.

\section{Abstract}

Background: Serosurveillance study focusing on antibodies against SARS-CoV2 among the Covid19 cases can add value in the scientific knowledge $\&$ help in formulating valid predictions regarding immunity status in the post-covid period. Objectives: To estimate seropositivity among covid19 cases and to identify various factors affecting seropositivity. Methods: During second half of October 2020, a population based serosurvey on Covid19 cases was carried out in Ahmedabad. Covid-Kavach test kits were used and estimated seroprevalence was compared with available demographic and covid19 case related parameters to identify factors affecting seropositivity in the post-covid period. Simple proportions and Z-test were used as appropriate. Results: As on October 2020, the sero-positivity among Covid19 cases in Ahmedabad was 54.51\% [95\% Confidence Interval (CI) $52.14-56.86 \%$ ]. Females have higher positivity $(54.78 \%)$ as compared to males $(54.30 \%)$ but the difference was statistically not significant $(Z=0.19, P=0.84)$. Among children and elderly, the positivity is high and from young adults to elderly the seropositivity has an increasing trend. Severity of clinical illness and longer duration of hospitalization are associated with higher seropositivity. Conclusion: With $54.51 \%$ seropositivity among covid19 cases, it is clear that all the covid19 cases may not have developed IgG antibodies, have undetectable level or might have disappeared during the postcovid period. Comparison of seropositivity with age group and clinical case details clearly suggest close correlation with the severity of clinical symptoms. The seronegative cases indicate the need for further in-depth scientific research to identify the factors affecting immunity and to uncover the reasons behind the same.

\section{Keywords}

COVID-19 cases; Immunity; IgG Antibodies; SARS-CoV2; Seroprevalence

\section{Introduction}

Covid19, the disease caused by SARS-CoV2, spread across the world during 2020. $(1,2)$ In view of the large number of asymptomatic cases, as also suggested by WHO, the indirect estimation of actual cases is crucial in assessing the true extent of the spread of SARS-CoV2. $(3,4)$ Sero- surveillance identifies the proportion of asymptomatic or subclinical infection and helps in understanding the disease dynamics in a better way. $(5,6)$ This in turn helps in planning an appropriate public health response. Multiple sero-surveillance studies conducted during the pandemic have focused on antibodies against SARS-CoV2 in the general population. $(7,8)$ Sero-surveillance studies among 

covid19 cases can give scientific insight. Comparison of seropositivity among cases can add value in the scientific knowledge \& help in formulating valid predictions regarding immunity status in the post-covid period.

Ahmedabad city with approximately 7 million people was one of the earliest cities to witness the high case load in the initial months of the pandemic in India. We carried out a population based sero-surveillance during the second half of October'20. In this sero-surveillance, apart from the general population, covid19 cases were also included as an additional category along with health care workers and contacts of cases. This article covers and describes only the "Cases" component of the sero-surveillance study.

\section{Aims \& Objectives}

1. To estimate the seroprevalence among COVID-19 cases

2. To correlate the seropositivity with various demographic and other factors

\section{Material \& Methods}

With the objective of estimating the proportion of population exposed to SARS-CoV-2, Indian Council of Medical Research (ICMR) had issued directives for conducting IgG antibody based sero-surveys to monitor the pandemic, understand its progression and to take appropriate corrective public health measures. The authority of the Ahmedabad Municipal Corporation (AMC), from the state of Gujarat, INDIA, already completed two large scale population based serosurveillance for IgG antibodies against SARS-CoV-2 during June \& August 2020. A repeat population based serosurvey, 3rd serosurvey in Ahmedabad was carried out during the second half of October 2020. The sample size for the general population category was calculated based on the results of the previous studies using the population based stratified sampling. Confirm cases of Covid-19 were covered separately along with population based serosurvey. Sample size for the "Case" category was decided as atleast $10 \%$ of the general population sample targets, and covered separately over and above the general population sample. Thus, the "case" selection was unrelated to the covid19 cases and based on population proportion. To enroll sufficient number of cases from different time period since the pandemic, UPHC medical officers were advised to enroll cases registered during different months since the beginning of the pandemic. 'WHO' has given the definition of a case of covid19, including the suspected, probable and confirmed case.(9) We used the confirmed case definition of covid19 for the purpose of our study. "Covid Kavach" (Anti-SARS CoV-2 IgG Antibody Detection capture ELISA) kit developed and manufactured by Zydus Diagnostics, validated by National Institute of Virology, Pune, India and approved for use by ICMR was used for the purpose of this study. Covid-Kavach has sensitivity of $92.37 \%$ and specificity of $97.9 \%$ as per the validation reports.(10) Thus, with a high level of sensitivity and specificity, the results received through this testing kit is quite reliable. The manufacturer reported no cross-reactivity with other viruses in the serum from RTPCR confirmed patients of various other infections. Testing procedures were followed as per the manufacturer's instructions. To reduce the sample rejection rate, SST-Gel Vacutee were used for the collection of blood samples.

The primary health care network in Ahmedabad city includes 75 Urban Primary Health Centres (UPHCs) within 48 wards, across 7 zones. Since the UPHCs are functional units for the covid19 case management, these 75 UPHCs have the details of all the reported cases from their area. Based on the calculated sample size, COVID-19 cases of either sex diagnosed at any point of time, who gave informed written consent, were enrolled as 'case'. However, so far as possible, an effort was specifically made to cover a wide variety of cases from different time period since the beginning of the pandemic, of different age-group (with atleast $10 \%$ of cases from $<18$ years and $>60$ years age) from different localities within the field area of the UPHC.

The study was carried out after the approval of ethics committee of AMC MET Medical College. A written informed consent was taken from all the participants before enrollment $\&$ in case of minors, an assent with informed written consent of their parent/guardian was taken for the purpose of the study. Strict confidentiality was ensured at all the levels. For the purpose of testing and standardization, only those laboratories with national level accreditation and state of the art facilities and equipment were approved for testing the samples. Microsoft Excel and Epi-Info was used for the purpose of data management. In-depth analysis of the data was carried out with focus on the seropositivity and its correlation among cases with various demographic and other factors. Simple proportions and appropriate statistical tests were used wherever required.

\section{Results}

A total of 1711 (745 female, 966 male) samples were collected out of which 3 samples were rejected (2 Female \& 1 Male). Results were thus available for 1708 cases. From these results 758 (44.38\%) were negative and 19 (1.11\%) had indeterminate results. Thus, a total of 931 results were positive for the IgG antibodies giving an overall positivity of $54.51 \%$ [ $95 \%$ Confidence Interval (Cl) 52.14-56.86\%].

Detailed analysis of 1708 case (Table-1) show that there were 743 females and 965 males for whom results were available. A total of 407 samples were positive among female giving a positivity rate of $54.78 \%$ [95\% Cl $51.18-$ $58.32 \%$ ] whereas 524 samples were positive among male giving a positivity of $54.30 \%$ [ $95 \% \mathrm{Cl} 51.15-57.42 \%]$ This 
difference between two sex group is statistically not significant $(\mathrm{Z}=0.19, \mathrm{P}=0.84)$.

The age distribution of the cases typically follows ageheaping bias at 5 years gap (data not shown, only grouped data shown in data table) as the age of the enrolled individual cases were not verified with any official document. The age of the cases ranged from 3-93 years with a mode of 40, median of 40 and an average of $41.67+16.23$ years. Considering the sero-positive cases, the mean age for females is $44.82+15.80$ years where as that of male is $44.76+17.06$ years.

The age group wise analysis of positivity among cases (Figure-1) shows that the lowest positivity is for 20-29 years i.e., $39.14 \%$. Children had higher seropositivity than young adults. From young adults to elderly the seropositivity gradually increases from around $40 \%$ to around $70 \%$. On both the extremes of age range, i.e., in children and elderly, the positivity is high as compared to the young adults. When the age group wise positivity is further bifurcated according to sex, the trend almost remains similar with variations at both the extremes of the age. Analyzing the zone wise seropositivity, the highest positivity was recorded in South Zone (71.67\%) and the lowest in the South West Zone (40.15\%). All the other zones recorded seroprevalence among cases in a narrow range of $51.88 \%$ to $58.55 \%$.

Looking into details of patient management (Figure-2) cases requiring hospital admission had higher seropositivity $(72.31 \%)$ as compared to cases managed at Covid Care Centre (CCC) $(52.38 \%)$ and cases managed at home had the lowest seropositivity (49.41\%). Among the hospitalised cases, patients on ventilator had the highest seropositivity (100\%) as compared to patients requiring Oxygen (81.58\%) with cases not requiring oxygen had the lowest seropositivity (70.91\%). According to duration of hospitalization the seropositivity for $<1$ week, 1-2 week, 23 week \& >3 week were 62.35\%, 73.30\%, 75.76\% \& 100\% respectively.

We also analyzed the time gap between the date of sero sample collection to the date of diagnosis of Covid19. While comparing the time since diagnosis in months, (Figure-3), IgG antibodies were detected in the range of $50-55 \%$ during the first 4 months of time-gap. For 5 months and 6 months it was $69.70 \%$ \& $61.54 \%$.

\section{Discussion}

General immune response after any viral infection has already been documented. However, as Covid19 is a novel viral infection, the immune response during and after covid19 infection is still largely evolving. $(11,12)$ The present study on the seropositivity among cases is among the few serological studies from India, exclusively covering the cases of covid-19 cases with a large sample.

Seropositive covid19 cases are the laboratory confirmed cases, who demonstrate IgG antibodies after the infection. This is a direct evidence of immune response as a result of their infection. While seropositive cases directly indicate proportion of cases who have acquired immune response, the seronegative cases indicate the proportion of cases who did not demonstrate IgG antibodies inspite of having a confirmed infection status in the past. "What are the factors affecting this seropositivity?" is the real question which the experts are trying to answer with scientific evidences. The present study also tries to highlight the proportion of cases with IgG antibodies and its correlation with the duration, severity and time-gap since diagnosis, if any.

It is quite obvious that one would expect all the confirmed cases of Covid19 to have IgG antibodies against the disease agent, SARS-CoV2. However, based on our findings with an average seropositivity of $54.51 \%[95 \% \mathrm{Cl}$ 52.14-56.86\%] among cases, it can be said that majority of the cases demonstrate presence of IgG antibodies after the infection with SARS-CoV2. However, it also implies that the remaining $45.49 \%$ have either not developed the antibodies, have antibodies but in undetectable proportion or the antibodies have disappeared, after their development, during the post-covid period. The reasons for seronegative cases require further in-depth scientific research to identify the factors affecting immunity and to uncover the reasons behind the same.

Among the cases, females have higher positivity (54.78\%) as compared to males (54.30\%) but the difference was statistically not significant $(\mathrm{Z}=0.19, \mathrm{P}=0.84)$. This finding signifies that the difference is by chance and the factors affecting immunity seems to be affecting both the biological groups equally. Similar is the finding by other studies, where the difference between the two sex groups is statistically not significant. $(13,14)$

Since the sample size for cases was calculated based on the population proportion (independent of the cases recorded from each UPHC/Ward), the proportion of seropositive cases should not differ much. Although most zones have seropositive proportion between 51.88$58.55 \%$, the reasons for low seropositivity in West Zone (40.15\%) and high seropositivity in South Zone (71.67\%) are not clear. This may indicate that there may be multiple other factors affecting positivity in covid19 cases \& require further analysis for other factors affecting seropositivity.

Contrary to the general belief that any case of viral disease will have antibodies against the virus in the immediate post-infection period, as per our study the seropositivity among covid19 cases was not $100 \%$. So, it was important to identify the reasons for the same and analyse data to identify the relationship of the factors affecting the seropositivity. There are already documented evidences that the severity of the clinical symptoms affects the immune response. Duration of hospital stay, need for Oxygen / Bipap / Ventilator as part of case management as well as place of case management (home / Covid Care Centre (CCC) / hospitalization) are all directly related to 
the severity of clinical symptoms. We tried to analyse available details of the covid19 cases on these parameters to check this association.

Looking at the age-group wise seropositivity (Figure-1), children and adolescent have the higher seropositivity. Most children and young adolescents are more likely to have mild clinical illness which may be due to their strong immune response which leads to development of IgG antibodies in the immediate post-covid period. On the other hand, the seropositivity of about $40 \%$ in $20-29$ years age group adults indicate that less than half from the young adult age group demonstrate IgG antibodies in the post-covid period. It also shows that the positivity has increasing trend as the age group increases from young adults to elderly. There are also scientific evidences which show that the elderly people are more likely to have symptoms, have more severe symptoms and their period of clinical symptoms stays for longer duration as compared to the young adults. $(15,16,17,18,19,20)$ These differences in the clinical symptoms may be the reason behind the higher seropositivity among elderly as compared to the young adults.

Available evidences suggest that the percent seroconversion in asymptomatic cases is low. $(21,22)$ On verifying this fact with our data, it was found that those requiring hospital admission demonstrated higher seropositivity than those cases who were isolated at home. The patient management principle required for these cases also showed that as the severity of case increase (from not requiring oxygen, requiring oxygen, Bipap to ventilator) the proportion of seropositive cases increases. Even the duration of hospitalization showed that as the duration of hospitalization increases the proportion of seropositive cases increase and all cases requiring hospital admission beyond 3 weeks were seropositive.

It has been documented that onset of symptoms should be preferred rather than the date of diagnosis for consideration of this time-gap.(16) However, due to higher reliability of data, we preferred comparing the date of diagnosis over the onset of symptoms. Comparing the proportion of seropositive cases with time since diagnosis (in months) (Figure-3), we observed that the seropositivity stays between $50-55 \%$ during the first 4 months of time since diagnosis. The higher seropositivity for $5 \& 6$ months from diagnosis coincides with the first peak of cases in the city. The relationship of seropositivity with the time since diagnosis is not very clear and require detailed scientific inquiry to understand the dynamics of immune reaction in the post covid period.

\section{Conclusion}

As on October 2020 , with $54.51 \%$ seropositivity among covid19 cases, it is clear that many covid19 cases may not have developed IgG antibodies, have undetectable level or might have disappeared during the post-covid period.
Sex wise difference in seropositivity is statistically not significant. Both the extremes of age show higher seropositivity and from young adults to elderly the proportion of positivity shows increasing trend. The severity of clinical symptom is clearly related with more pronounced immune response. The seronegative cases indicate the need for further in-depth scientific research to identify the factors affecting immunity and to uncover the reasons behind the same.

\section{Recommendation}

All the confirm cases of Covid19 may not have demonstrable IgG Antibodies. Seronegative cases indicate the need for further scientific research

\section{Limitation of the study}

We have collected limited demographic details of the enrolled Covid19 cases so that only limited analysis on serosurveillance result was possible. The limitations of the testing kit "Covid-Kavach" automatically applies to the findings of our study.

\section{Authors Contribution}

OP, JS, SV \& AS conceptualized the study. BS, DM and their team under the guidance of OP planned and carried out the serosurvey. Testing of the samples and reporting was managed by MK and her team. Data analysis was carried out by JS, SV, AS. The statistical analysis was done by HT. Primary manuscript was prepared by OP and JS and equally contributed by all the other coauthors. All authors contributed to the interpretation of data and approved the final manuscript after critical review.

\section{Acknowledgement}

We are extremely thankful to respected Dr. Rajiv Kumar Gupta, IAS (Additional Chief Secretary, Government of Gujarat) and Mr. Mukesh Kumar, IAS (Municipal Commissioner, Ahmedabad) for their whole hearted support. We acknowledge the full support from the field level health care workers (Corona warriors) who put in great efforts to perform their duties as well as sample collection after informed written consent particularly in a covid19 pandemic situation. All the Zonal Deputy Health Officers, Deputy Health Officer (Epidemic), Assistant Health Officers and Medical officers of the UPHCs extended full support in conducting the serosurveillance. We are thankful to all the medical and paramedical support staff posted at the laboratories for their contribution in timely testing of the samples with accuracy and quality. Finally, we are indebted to all the participants whose willingness and support has generated the muchdesired data for the study.

\section{References}

1. Munster VJ, Koopmans M, van Doremalen N, van Riel D, de Wit E. A Novel Coronavirus Emerging in China - Key Questions for Impact Assessment. N Engl J Med. 2020;382(8):692-694

2. Li, Q., Guan, X., Wu, P., Wang, X., Zhou, L., Tong, Y., Ren, R., Leung, K., Lau, E., Wong, J. Y., Xing, X., Xiang, N., Wu, Y., Li, C., Chen, Q., Li, D., Liu, T., Zhao, J., Liu, M., Tu, W., ... Feng, Z. Early Transmission Dynamics in 
INDIAN JOURNAL OF COMMUNITY HEALTH / VOL 33 / ISSUE NO 02 / APR- JUN 2021 Wuhan, China, of Novel Coronavirus-Infected Pneumonia. The New England journal of medicine, 382(13), 1199-1207.

3. GeurtsvanKessel, C. H., Okba, N., Igloi, Z., Bogers, S., Embregts, C., Laksono, B. M., Leijten, L., Rokx, C., Rijnders, B., Rahamat-Langendoen, J., van den Akker, J., van Kampen, J., van der Eijk, A. A., van Binnendijk, R. S., Haagmans, B., \& Koopmans, M. (2020). An evaluation of COVID19 serological assays informs future diagnostics and exposure assessment. Nature communications, 11(1), 3436. https://doi.org/10.1038/s41467-020-17317-y

4. World Health Organization. Population-based age-stratified seroepidemiological investigation protocol for COVID-19 virus infection, 17 March 2020. World Health Organization; 2020.

5. Hanage, William, Xueting Qiu, Lee Kennedy-Shaffer. Snowball Sampling Study Design for Serosurveys in the Early COVID-19 Pandemic (2020). Available from: https://nrs.harvard.edu/URN3:HUL.INSTREPOS:37363145 (Accessed on 25.06.2021)

6. Cohen J. Unprecedented nationwide blood studies seek to track U.S. coronavirus spread. Science. 2020 Apr 7 Available from: https://www.sciencemag.org/news/2020/04/unprecedentednationwide-blood-studies-seek-track-us-coronavirus-spread (Accessed on 25.06.2021)

7. $\mathrm{Xu}, \mathrm{X} ., \mathrm{Sun}, \mathrm{J.}, \mathrm{Nie}$, S. et al. Seroprevalence of immunoglobulin $\mathrm{M}$ and $\mathrm{G}$ antibodies against SARS-CoV-2 in China. Nat Med 26, 1193-1195 (2020). https://doi.org/10.1038/s41591-020-0949-6

8. Murhekar M V, Bhatnagar T, Selvaraju S, Rade K, Saravanakumar V, Wesley Vivian Thangaraj J, et al. Prevalence of SARS-CoV-2 infection in India: Findings from the national serosurvey, May-June 2020. Indian J Med Res. 2020;152:48-60. DOI: 10.4103/ijmr.IJMR_3290_20 PMID: 32952144 PMCID: PMC7853249

9. World Health Organization. Public health surveillance for COVID-19: interim guidance, 7 August 2020 [Internet]. Geneva; 2020 Aug. Available from: https://apps.who.int/iris/bitstream/handle/10665/333752/WHO2019-nCoV-SurveillanceGuidance-2020.7-

eng.pdf?sequence $=1 \&$ isAllowed $=y$ (Accessed on 25.06.2021)

10. Sapkal G, Shete-Aich A, Jain R, et al. Development of indigenous IgG ELISA for the detection of anti-SARS-CoV-2 IgG. Indian J Med Res. 2020;151(5):444-449. doi:10.4103/ijmr.IJMR_2232_20 PMID: 32611915 PMCID: PMC7530443

11. Chirathaworn C, Sripramote M, Chalongviriyalert P, et al. SARS-CoV-2 RNA shedding in recovered COVID-19 cases and the presence of antibodies against SARS-CoV-2 in recovered COVID-19 cases and close contacts, Thailand, April-June 2020. PLoS One. 2020;15(10):e0236905. Published 2020 Oct 29. doi:10.1371/journal.pone.0236905 PMID: 33119712 PMCID: PMC7595404

12. Martinez-Fierro, M. L., Ríos-Jasso, J., Garza-Veloz, I., Reyes-Veyna, L., Cerda-Luna, R. M., Duque-Jara, I., Galvan-Jimenez, M., RamirezHernandez, L. A., Morales-Esquivel, A., Ortiz-Castro, Y., GutierrezCamacho, J. R., Valdes-Aguayo, J. J., \& Vargas-Rodriguez, J. R. (2021). The role of close contacts of COVID-19 patients in the SARS-CoV-2
[Serosurveillance among...] | Prakash O et al transmission: an emphasis on the percentage of nonevaluated positivity in Mexico. American journal of infection control, 49(1), 15-20. https://doi.org/10.1016/j.ajic.2020.10.002

13. Bryant JE, Azman AS, Ferrari MJ, et al. Serology for SARS-CoV-2 Apprehensions, opportunities, and the path forward. Sci Immunol. 2020;5(47):eabc6347. doi:10.1126/sciimmunol.abc6347 PMID: 32430309

14. Stringhini S, Wisniak A, Piumatti G, et al. Seroprevalence of anti-SARSCoV-2 IgG antibodies in Geneva, Switzerland (SEROCoV-POP): a population-based study. Lancet. 2020;396(10247):313-319. doi:10.1016/S0140-6736(20)31304-0 PMID: 32534626 PMCID: PMC7289564

15. Wang, X., Guo, X., Xin, Q., Pan, Y., Hu, Y., Li, J., Chu, Y., Feng, Y., \& Wang, Q. (2020). Neutralizing Antibody Responses to Severe Acute Respiratory Syndrome Coronavirus 2 in Coronavirus Disease 2019 Inpatients and Convalescent Patients. Clinical infectious diseases : an official publication of the Infectious Diseases Society of America, 71(10), 26882694. https://doi.org/10.1093/cid/ciaa721

16. Hu WT, Howell JC, Ozturk T, et al. Antibody Profiles According to Mild or Severe SARS-CoV-2 Infection, Atlanta, Georgia, USA, 2020. Emerg Infect Dis. 2020;26(12):2974-2978. doi:10.3201/eid2612.203334 doi: 10.3201/eid2612.203334 PMID: 32857691 PMCID: PMC7706962

17. Okba NMA, Müller MA, Li W, et al. Severe Acute Respiratory Syndrome Coronavirus 2-Specific Antibody Responses in Coronavirus Disease Patients. Emerg Infect Dis. 2020;26(7):1478-1488. doi:10.3201/eid2607.200841 PMID: 32267220 PMCID: PMC7323511

18. Kellam $P$, Barclay $W$. The dynamics of humoral immune responses following SARS-CoV-2 infection and the potential for reinfection. J Gen Virol. 2020;101(8):791-797. doi:10.1099/jgv.0.001439 PMID: 32430094 PMCID: PMC7641391

19. Cervia C, Nilsson J, Zurbuchen $Y$, et al. Systemic and mucosal antibody responses specific to SARS-CoV-2 during mild versus severe COVID-19. J Allergy Clin Immunol. 2021;147(2):545-557.e9. doi:10.1016/j.jaci.2020.10.040 PMID: 33221383 PMCID: PMC7677074

20. Nikolai, L. A., Meyer, C. G., Kremsner, P. G., \& Velavan, T. P. (2020). Asymptomatic SARS Coronavirus 2 infection: Invisible yet invincible. International journal of infectious diseases: IJID : official publication of the International Society for Infectious Diseases, 100, 112-116. https://doi.org/10.1016/j.ijid.2020.08.076

21. Yongchen Z, Shen H, Wang X, et al. Different longitudinal patterns of nucleic acid and serology testing results based on disease severity of COVID-19 patients. Emerg Microbes Infect. 2020;9(1):833-836. doi:10.1080/22221751.2020.1756699 PMID: 32306864 PMCID: PMC7241531

22. Wellinghausen, N., Plonné, D., Voss, M., Ivanova, R., Frodl, R., \& Deininger, S. (2020). SARS-CoV-2-IgG response is different in COVID-19 outpatients and asymptomatic contact persons. Journal of clinical virology : the official publication of the Pan American Society for Clinical Virology, 130, 104542. https://doi.org/10.1016/j.jcv.2020.104542

\section{Tables}

TABLE 1 ANALYSIS OF COVID19 SERO-SURVEY POSITIVITY AMONG CASES

\begin{tabular}{|c|c|c|c|c|c|c|c|c|c|c|}
\hline \multicolumn{4}{|c|}{ Female } & \multicolumn{3}{|c|}{ Male } & \multicolumn{3}{|c|}{ Total } & \multirow[b]{2}{*}{$95 \% \mathrm{Cl}$} \\
\hline & Results & Positive & $\%$ Positivity & Results & Positive & \% Positivity & Results & Positive & \% Positivity & \\
\hline Total & 743 & 407 & 54.78 & 965 & 524 & 54.30 & 1708 & 931 & 54.51 & $52.14-56.86$ \\
\hline \multicolumn{11}{|c|}{ Age group } \\
\hline $0-9$ & 4 & 2 & 50.00 & 6 & 5 & 83.33 & 10 & 7 & 70.00 & $34.75-93.33$ \\
\hline $10-19$ & 49 & 23 & 46.94 & 59 & 26 & 44.07 & 108 & 49 & 45.37 & $35.76-55.24$ \\
\hline $20-29$ & 145 & 50 & 34.48 & 182 & 78 & 42.86 & 327 & 128 & 39.14 & $34.01-44.53$ \\
\hline $30-39$ & 154 & 78 & 50.65 & 226 & 108 & 47.79 & 380 & 186 & 48.95 & $43.96-53.96$ \\
\hline $40-49$ & 137 & 78 & 56.93 & 170 & 84 & 49.41 & 307 & 162 & 52.77 & $47.18-58.28$ \\
\hline $50-59$ & 130 & 92 & 70.77 & 154 & 101 & 65.58 & 284 & 193 & 67.96 & $62.19-73.35$ \\
\hline $60-69$ & 96 & 67 & 69.79 & 112 & 78 & 69.64 & 208 & 145 & 69.71 & $62.98-75.87$ \\
\hline $70-79$ & 23 & 13 & 56.52 & 49 & 39 & 79.59 & 72 & 52 & 72.22 & $60.41-82.14$ \\
\hline $80-89$ & 3 & 2 & 66.67 & 7 & 5 & 71.43 & 10 & 7 & 70.00 & $34.75-93.33$ \\
\hline $90-99$ & 2 & 2 & 100.00 & 0 & 0 & & 2 & 2 & 100.00 & $15.81-100.0$ \\
\hline \multicolumn{11}{|l|}{ Zone } \\
\hline CZ & 85 & 48 & 56.47 & 96 & 53 & 55.21 & 181 & 101 & 55.80 & $48.25-63.16$ \\
\hline EZ & 119 & 61 & 51.26 & 108 & 60 & 55.56 & 227 & 121 & 53.30 & $46.59-59.93$ \\
\hline
\end{tabular}




\begin{tabular}{|rrrrrrrrrrr|r|r|}
\hline & Results & Positive & \% Positivity & Results & Positive & \% Positivity & Results & Positive & \% Positivity & $95 \%$ Cl \\
\hline NWZ & 35 & 23 & 65.71 & 98 & 46 & 46.94 & 133 & 69 & 51.88 & $43.05-60.62$ \\
\hline NZ & 170 & 95 & 55.88 & 151 & 85 & 56.29 & 321 & 180 & 56.07 & $50.61-61.40$ \\
\hline SWZ & 64 & 32 & 50.00 & 88 & 57 & 64.77 & 152 & 89 & 58.55 & $50.29-66.48$ \\
\hline SZ & 123 & 84 & 68.29 & 170 & 126 & 74.12 & 293 & 210 & 71.67 & $66.14-76.76$ \\
\hline WZ & 147 & 64 & 43.54 & 254 & 97 & 38.19 & 401 & 161 & 40.15 & $35.47-45.02$ \\
\hline
\end{tabular}

\section{Figures}

\section{FIGURE 1 AGE GROUP WISE SEROPOSITIVITY AMONG CASES}

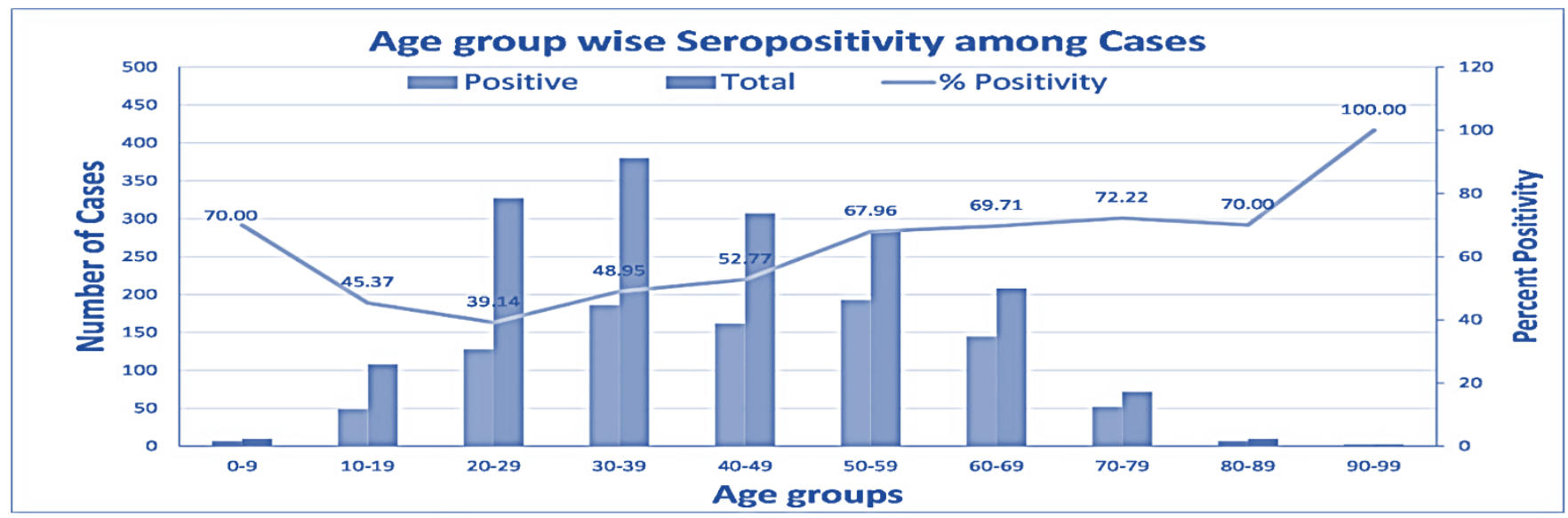

\section{FIGURE 2 SEROPOSITIVITY ACCORDING TO CASE MANAGEMENT DETAILS}

Percent Seropositivity according to Case Management details

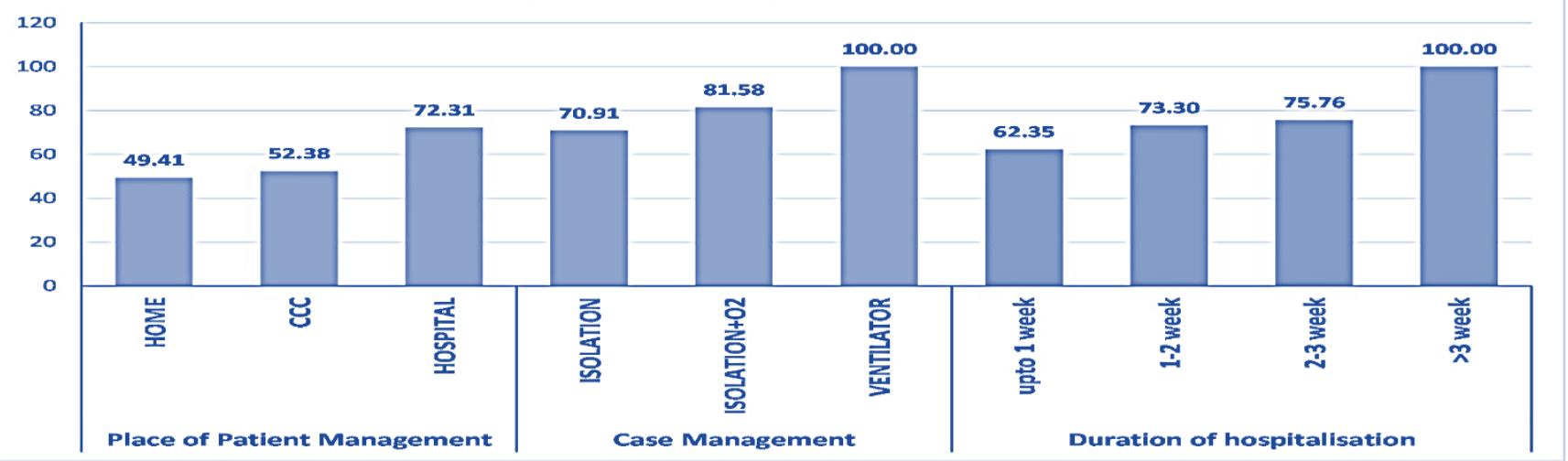

FIGURE 3 SEROPOSITIVITY AMONG CASES ACCORDING TO TIME SINCE DIAGNOSIS IN MONTHS

Percent Seropositivity among cases from time since diagnosis (months)

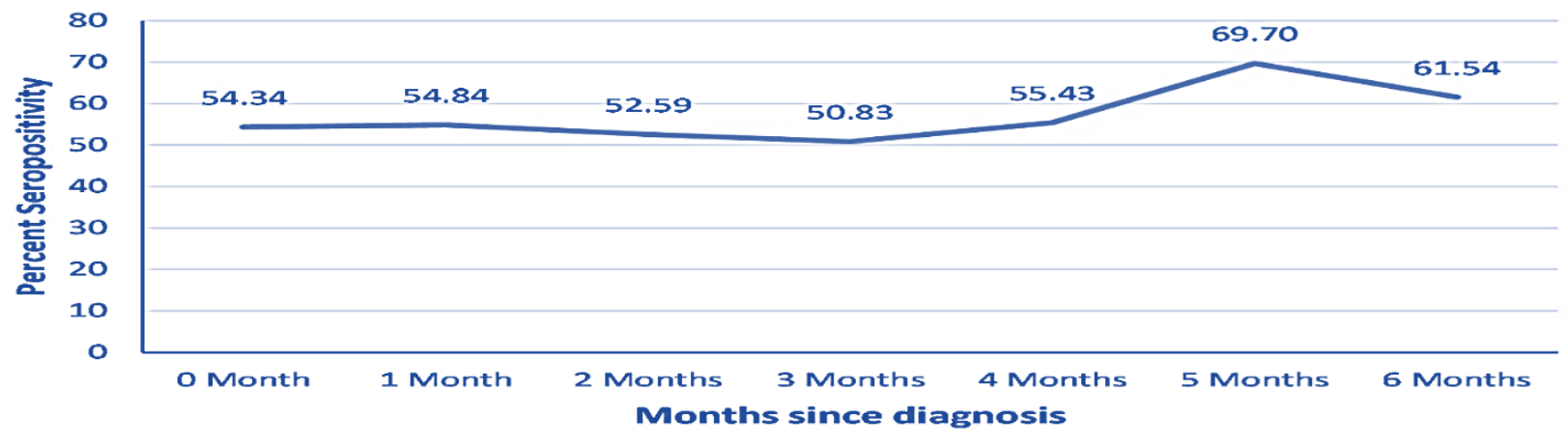

